\title{
Advances on geometric flux optical design method
}

\section{Ángel García-Botella, Antonio Álvarez Fernández- Balbuena, Daniel Vázquez}

Ángel García-Botella, Antonio Álvarez Fernández-Balbuena, Daniel Vázquez, "Advances on geometric flux optical design method," Proc. SPIE 10379, Nonimaging Optics: Efficient Design for Illumination and Solar Concentration XIV, 103790J (7 September 2017); doi: 10.1117/12.2281481

EDent: SPIE Optical Engineering + Applications, 2017, San Diego, California, United States 


\title{
Advances on geometric flux optical design method
}

\author{
Ángel García-Botella*a , Antonio Álvarez Fernández-Balbuena ${ }^{\mathrm{a}, \mathrm{b}}$, Daniel Vázquez ${ }^{\mathrm{b}}$ \\ a Dpto. de Ingeniería y Gestión Forestal y Ambiental, Universidad Politécnica de Madrid, E.T.S.I. de \\ Montes, Forestal y del Medio Natural, Ciudad Universitaria s/n, 28040 Madrid, (SPAIN) \\ ${ }^{\mathrm{b}}$ Dpto. de Óptica, Universidad Complutense de Madrid, Facultad de Óptica, Arcos de Jalón,

$$
\text { s/n, } 28037 \text { Madrid (SPAIN) }
$$

(*) ORCID: 0000-0003-1861-0352

\begin{abstract}
Nonimaging optics is focused on the study of methods to design concentrators or illuminators systems. It can be included in the area of photometry and radiometry and it is governed by the laws of geometrical optics. The field vector method, which starts with the definition of the irradiance vector $\mathbf{E}$, is one of the techniques used in nonimaging optics. Called "Geometrical flux vector" it has provide ideal designs. The main property of this model is, its ability to estimate how radiant energy is transferred by the optical system, from the concepts of field line, flux tube and pseudopotential surface, overcoming traditional raytrace methods. Nevertheless this model has been developed only at an academic level, where characteristic optical parameters are ideal not real and the studied geometries are simple. The main objective of the present paper is the application of the vector field method to the analysis and design of real concentration and illumination systems. We propose the development of a calculation tool for optical simulations by vector field, using algorithms based on Fermat s principle, as an alternative to traditional tools for optical simulations by raytrace, based on reflection and refraction law. This new tool provides, first, traditional simulations results - efficiency, illuminance/irradiance calculations, angular distribution of light- with lower computation time, photometrical information needs about a few tens of field lines, in comparison with million rays needed nowadays. On the other hand the tool will provides new information as vector field maps produced by the system, composed by field lines and quasipotential surfaces. We show our first results with the vector field simulation tool.
\end{abstract}

Keywords: Nonimaging optics, geometric flux, vector field

\section{INTRODUCTION}

The concept of vector flux field was first introduced as a photometrical theory by Gershun [1] and later developed by Moon [2]. The theory is based on the definition of the irradiance vector $\mathbf{J}$ produced by a Lambertian source at any point in the space $P$. The module of $\mathbf{J}$ is the maximum irradiance value at point $P$; the direction of the vector is the direction of the maximum irradiance at $P$, the projection of the $\mathbf{J}$ vector upon a plane is directly the irradiance on that plane. During last decades vector flux field formalism has been applied to several problems in optics. In the $70^{\text {th }}$ decade of last century, Withrop [3] applied it to the study of propagation of structural information; at the end of that decade, Winston [4], [5] applied it successfully to the study of concentrators, showing that reflective concentrators with the geometry of the field lines achieve the theoretical limit of concentration. Later, Winston et al. [6] developed this method and others to build a new topic called Nonimaging Optics. Recently, vector flux field has been used to design so-called Hyperparabolic Concentrators (HPC) [7], which extend the Compound Parabolic Concentrator (CPC) to multiple reflections and approaches to the thermodynamic limit of concentration.

The mathematical theory of vector flux field was developed by Gershun [1], Fock [8], Moon [2] and others at the first half of last centrury. They concluded that vector flux field is solenoidal for points outside the sources and in general it is not an irrotational field

$$
\operatorname{curl} \mathrm{J} \neq 0 \text {. }
$$

\footnotetext{
Nonimaging Optics: Efficient Design for Illumination and Solar Concentration XIV, edited by Roland Winston, Sarah R. Kurtz, Proc. of SPIE Vol. 10379, 103790J

(C) 2017 SPIE - CCC code: $0277-786 X / 17 / \$ 18 \cdot$ doi: 10.1117/12.2281481
} 
Nevertheless there are many practical situations, 2 dimensional fields or rotational symmetric fields, for which $\mathbf{J}$ vector is orthogonal to its curl. Then pseudopotential surfaces can be defined as surfaces orthogonal to the $\mathbf{J}$ vector. Field lines produced by a Lambertian strip, are a family of confocal hyperbolas with foci at $A$ and $A$ ', and corresponding pseudopotential lines, a family of confocal elipses with foci at $A$ and $A^{\prime}$. For a Lambertian disk, the pseudopotential surfaces can be obtained by rotational symmetry of these lines. Another consequence of the application of field theory to optical design is that refractive elements with the geometry of pseudopotential lines produce ideal concentrators [9].

This theoretical approach to the vector irradiance theory nowadays is focused, on what can be said, "academicals results". No relevant practical results have been obtained, and the capabilities of this new tool have not been developed yet. The main objective of the present paper is the application of the vector field method to the analysis and design of real concentration and illumination systems.

This objective requires the implementation of real models for the optical parameters, reflectance and transmittance in the calculation of the field vector, as well as the study of the field produced by non- Lambertian sources and complex geometries. The implementation of parameters and sources will be done first for optical systems with only one component - reflexive/refractive- and later it will be generalized for $\mathrm{N}$ optical components. About the geometries, the work will start with the study of 2D geometries, and later it will be generalized to 3D systems.

We propose the development of a calculation tool for optical simulations by vector field, using algorithms based on Fermat`s principle, as an alternative to traditional tools for optical simulations by raytrace, based on reflection and refraction law. This new tool will provides, first, traditional simulations results - efficiency, illuminance/irradiance calculations, angular distribution of light- with lower computation time, photometrical information needs about a few tens of field lines, in comparison with million rays needed nowadays. On the other hand the tool will provides new information as vector field maps produced by the system, composed by field lines and quasipotential surfaces. The project is centered in to obtain and analyze these field maps and its application in the design of real optical systems. As consequence of this new simulation scheme, new optimization schemes will be analyzed. As result, we propose the development of the tool, first for 2D geometries and after to 3D.

\subsection{Simple introductory examples}

As a first step, to advance in this great objective we can study simple systems and analyse first comparisons between geometrical flux calculations and raytrace calculations to check the viability of the proposal. As first example we study the irradiance distribution produced in a plane at $80 \mathrm{~mm}$ height of two adjacent lambertian strip sources of 20mm length, the separation between two sources is $140 \mathrm{~mm}$. Figure 1 shows the comparisons between geometrical flux vector calculations and raytrace calculations. For these simple calculations geometrical flux provides exact solution, while raytrace needs to increase the number of rays to reduce the signal to noise ratio.

As a second example we study irradiance distribution produced by a Lambertian strip and a linear perfectly diffuse reflector, the length of the lambertian strip is $20 \mathrm{~mm}$, the diffuse reflector has a slope of $45^{\circ}$ and he irradiance profile as been calculated at $75 \mathrm{~mm}$ to the strip. Figure 2 shows the irradiance profile obtained using geometrical flux calculations and raytrace calculations, the figure shows too the irradiance profile of the same configuration without diffuse reflector, to compare different situations and to show the asymmetry produced by the reflector in the irradiance profile.

To conclude these introductory examples we study the same configuration of example 2, but instead of use a diffuse reflector we use a perfect specular reflector, which forms perfect symmetric image of the lambertian strip source, then it is easy to calculate the irradiance distribution using vector flux formalism. Figure 3 shows the comparison between irradiance distribution calculated using vector flux formalism and raytrace calculations, the figure shoes great agreement in the maximum location and in the tail of the profile, as expected.

These examples shows the viability to study geometric vector flux calculations, as an alternative to standard raytrace calculations, of course there are a lot of problems and challenge to overcome to arrive to the objective. The next paragraphs of the paper show the next steps on that way. 


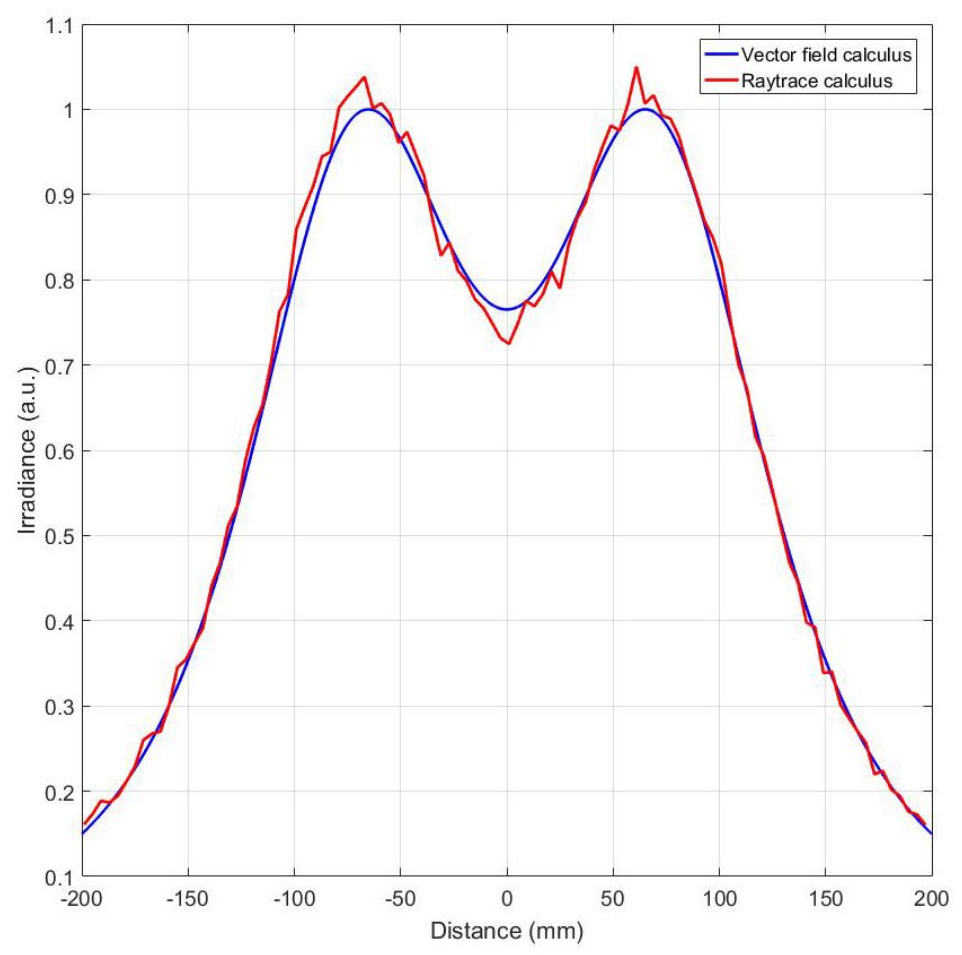

Figure 1. Comparison between geometrical flux vector calculus and raytrace. Configuration with two 2D adjacents lambertian strip source.

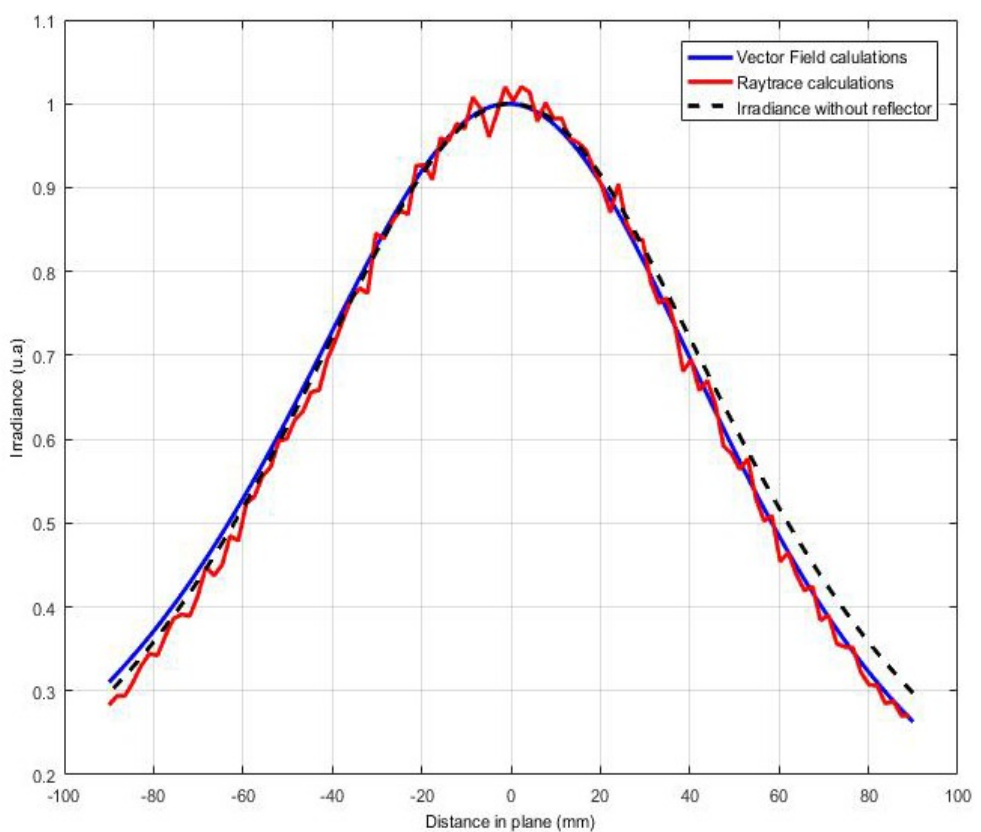

Figure 2. Comparison between geometrical flux vector and raytrace calculus. Configuration with 2D lambertian strip source and a linear diffuse reflector. 


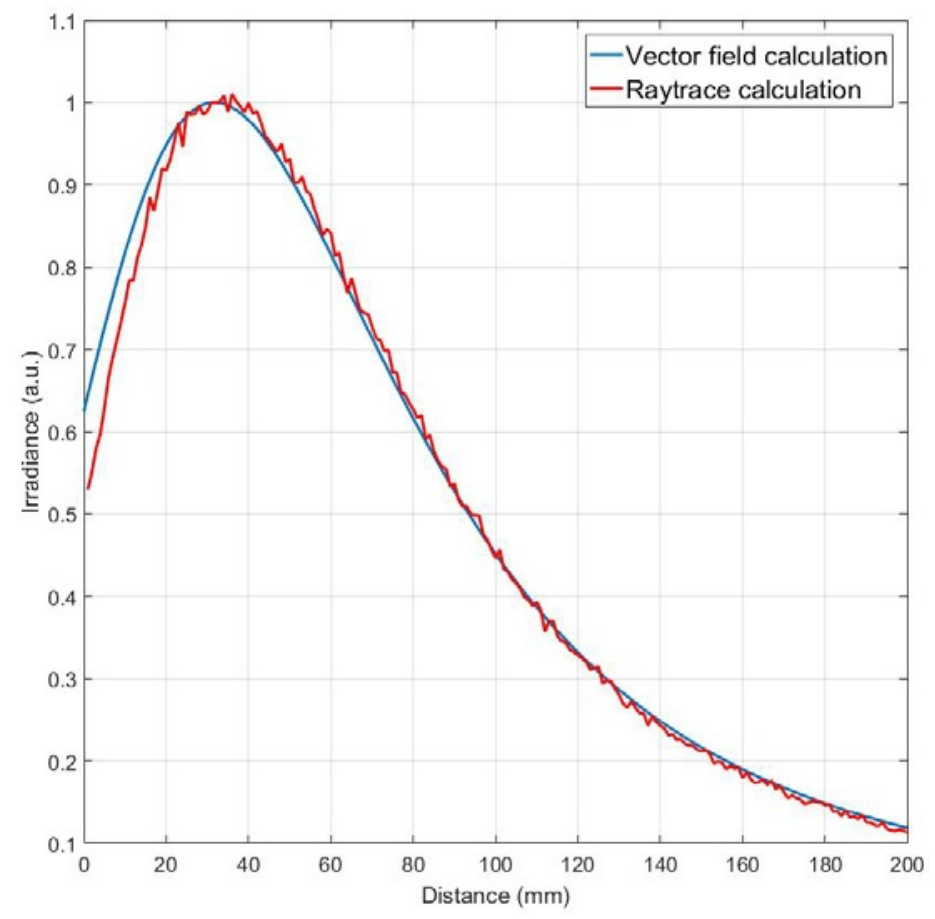

Figure 3. Comparison between geometrical flux vector calculus and raytrace. Configuration with 2D lambertian strip source and a linear specular reflector.

\section{FIELD LINES ALGORITHMS TO GEOMETRIC FLUX VECTOR CALCULATIONS}

As a principal step on the proposed work it is possible to identify the need to use global algorithms to evaluate the geometric flux vector at each point in the space. It is necessary an algorithm able to be used in 2D and 3D calculations, able to study reflexive, refractive and diffuse materials, able to study systems with $\mathrm{N}$ optical surfaces. In fact it must be a fundamental algorithm to cover all possible configurations. We propose to use two algorithms to calculate geometrical flux vector at any point, one algorithm to obtain the direction of the $\boldsymbol{J}$ vector and other algorithm to obtain the modulus of $\boldsymbol{J}$ vector. Both algorithms are based of field line calculations; first we can obtain the direction of the $\boldsymbol{J}$ vector at point $P$, as the tangent direction of field line at $P$, second we can calculate the modulus of $\boldsymbol{J}$ vector as a proportional relation with the density of field lines at $P$. Table 1 schematizes this situation.

Table 1. Schematic approach to field line algorithm.

\section{FIELD LINES ALGORITHMS}

$\boldsymbol{J}$ direction at point $P \rightarrow$ Tangent direction to field line at point $P$

$\boldsymbol{J}$ modulus at point $P \rightarrow$ Density of field lines at point $P$ 
Then it is clear that we must center our effort in field lines calculations, from which we can evaluate $\boldsymbol{J}$ vector. For centered symmetrical designs it is well stablished that field lines lies along contours with constant optical path length difference between edge rays [6]. Which is the same, the field line direction at point $P$ bisects the edge rays of the source. What it is clear is to compute the field lines we need to compute edge rays of the source, and then it is possible using Fermat principle.

\subsection{Source, lens, detector system}

To advance in the development of the computation algorithm we have prove the basic axisymmetric system composed by a Lambertian strip source, a plane-convex lens and a detector. We have implemented the algorithm based on Fermat's principle explained previously, and we have compute irradiance as proportional to the density of field lines. Figure 4 shows a sketch of the analyzed system, we use a source of $10 \mathrm{~mm}$ length, a plano convex lens placed 300mm to the source (plane surface), the convex surface has radio $R=150 \mathrm{~mm}$ and we have placed the detector at $1000 \mathrm{~mm}$ to the source. We have compared the results of vector field computations with raytrace computation. Figure 5 shows the comparison between vector field irradiance profile and raytrace irradiance profile. This configuration shows a minimum irradiance at the center of the profile and maximums about $25 \mathrm{~mm}$ to the center in the detector plane. Figure shows good agreement between vector field and raytrace computations, nevertheless vector field has two fundamental advantages, first vector field computation shows smooth profiles instead of noisy profiles obtained by raytrace computations, second computation time, raytrace needs great computation time to obtain smooth profiles similar those obtained by vector field computations.

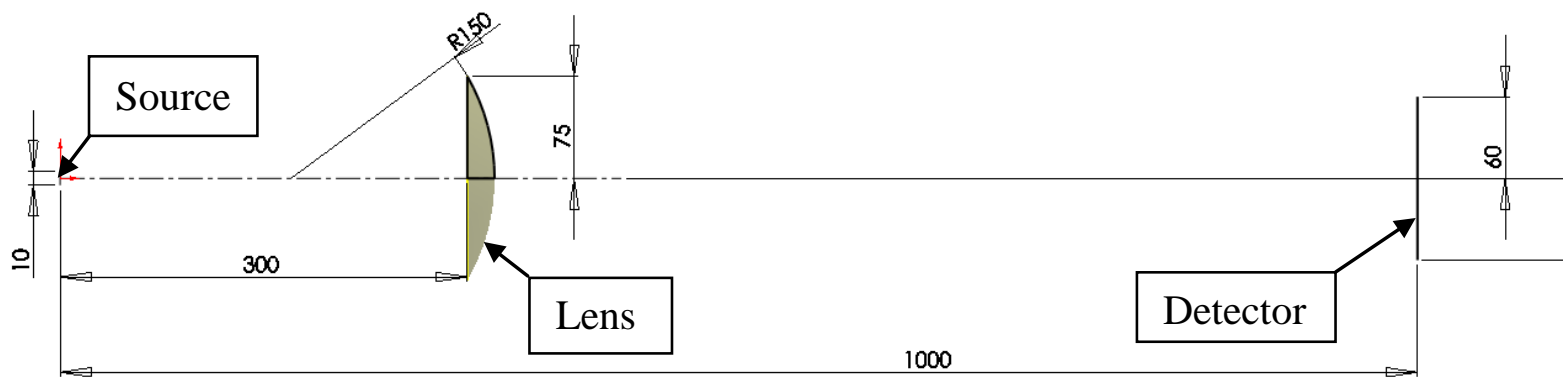

Figure 4. Sketch of the system analyzed by vector field calculations

We have study the same system for several distance of detector; results are showed in figure 6 . The agreement between vector field and raytrace calculations remains unaltered for different configurations of the system. Then as conclusion, the next step in our development will be to build a demo of simulation software for this simple system composed by a source a lens and a detector 


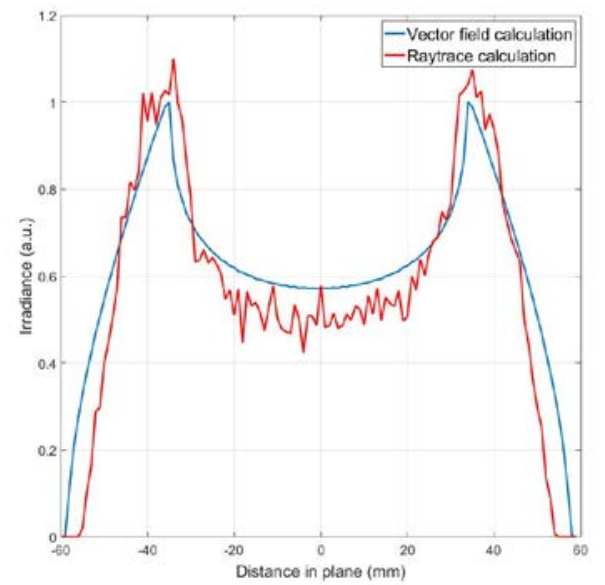

Figure 5. Comparison between vector field calculations and raytrace calculations, for a source, lens, detector system.
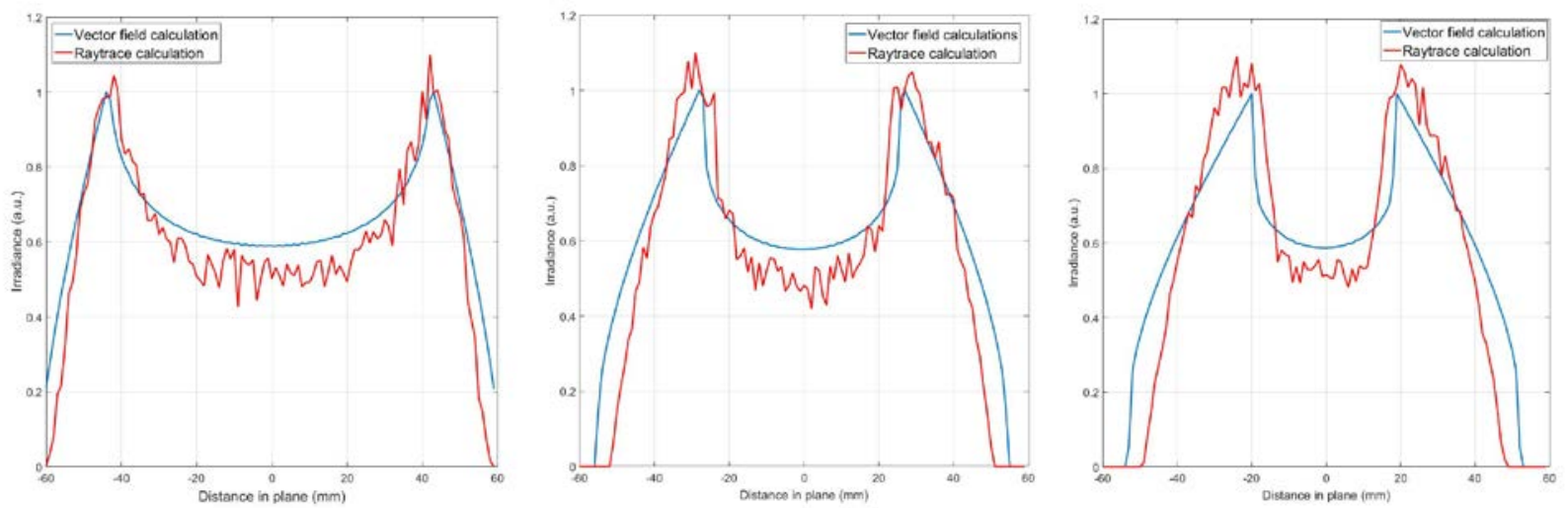

Figure 6. Comparison for distances between lens and detector of 850, 1150 and 1300mm.

For the same lens system, figure 7 shows typical irradiance distribution with a central maximum, in this case the parameters of the system are, source of $28 \mathrm{~mm}$ length, plano convex lens placed $450 \mathrm{~mm}$, radius of lens $150 \mathrm{~mm}$ and detector at $950 \mathrm{~mm}$ to the lens. The figure shows also good agreement between vector field calculations of irradiance at detector and raytrace calculations.

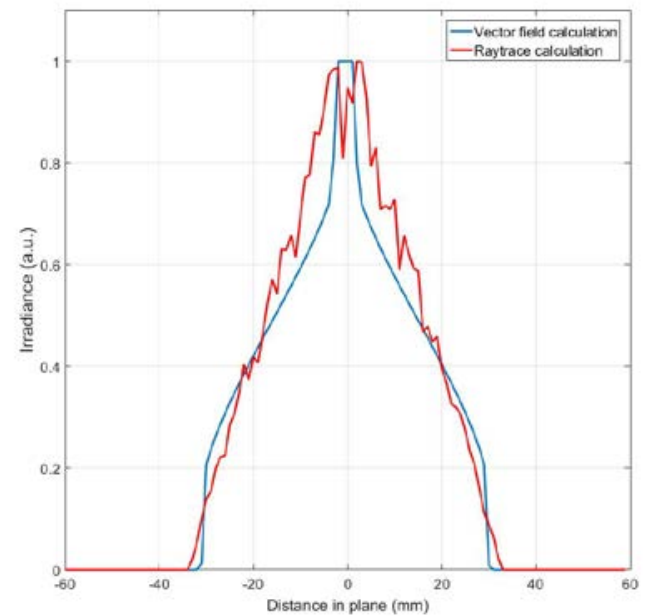

Figure 7. Comparison for a typical irradiance distribution with central maximum 


\subsection{Reflector, source, detector system}

As a second basic optical system, we have analyzed the behaviour of vector field algorithm for a system composed by a spherical reflector, a lamberitan source and a detector. The main difference between this system and previous one, is that in this case the irradiance distribution are produced by direct contribution $I_{d}$ to the source and reflected contribution $I_{r}$, in our analisys we have consider the reflectance $R$ of the reflector, then the irradiance in this case can be expresed as

$$
I=I_{d}+R I_{r} .
$$

Figure 8 shows a sketch of the reflector system, the design parameters are quite similar to the lens system, source length, reflector position, reflector radius, reflectance of reflector and detector position.

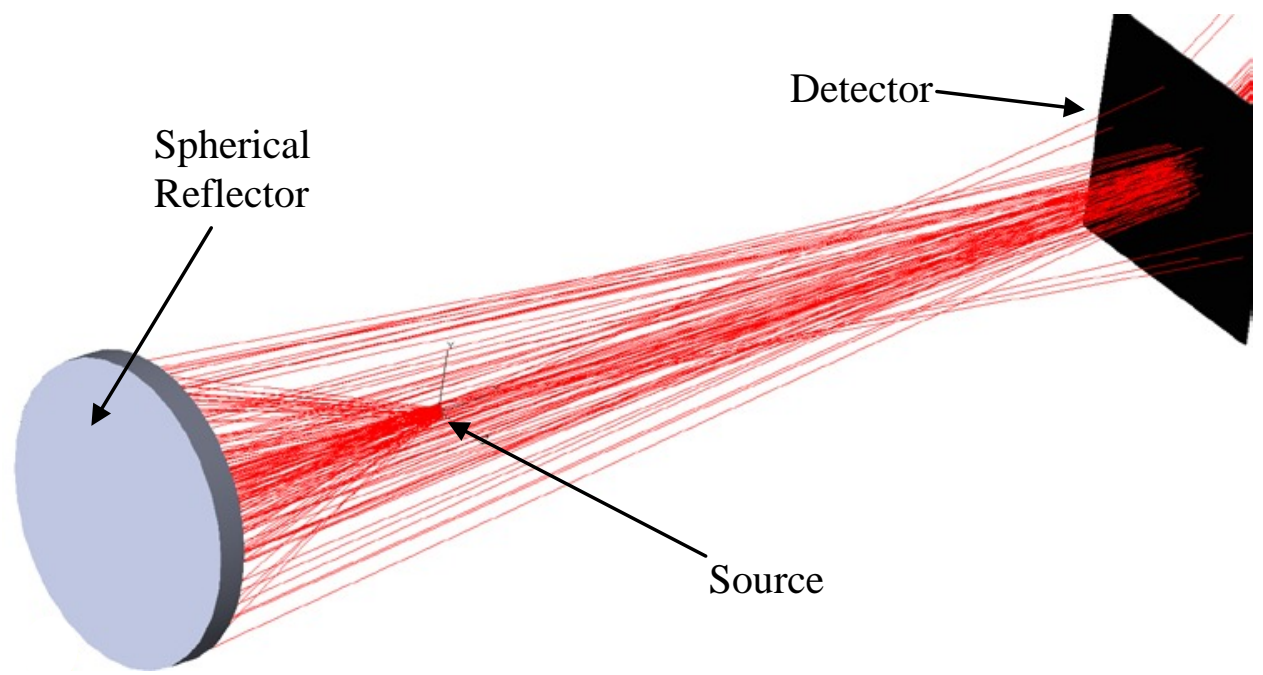

Figure 8. Sketch of the reflector, source and detector system

For this basic axisymmetric system we have built the algorithm to calculate irradiance distribution from vector field calculation and compare obtained results with raytrace calculus. We have done those task for several configurations of reflector system. Figure 9a shows irradiance distribution comparisons for reflector system with $8 \mathrm{~mm}$ source length, vertex of reflector at $190 \mathrm{~mm}$ to the source, radius of reflector $300 \mathrm{~mm}$, detector at $450 \mathrm{~mm}$ to the source and reflectance of reflector 0.9 , while figure $9 \mathrm{~b}$ shows the comparison for a system with source length of $12 \mathrm{~mm}$, vertex of reflector at $80 \mathrm{~mm}$ to the source, radius of reflector $170 \mathrm{~mm}$, detector at $500 \mathrm{~mm}$ to de source and reflectance of 0.9 .

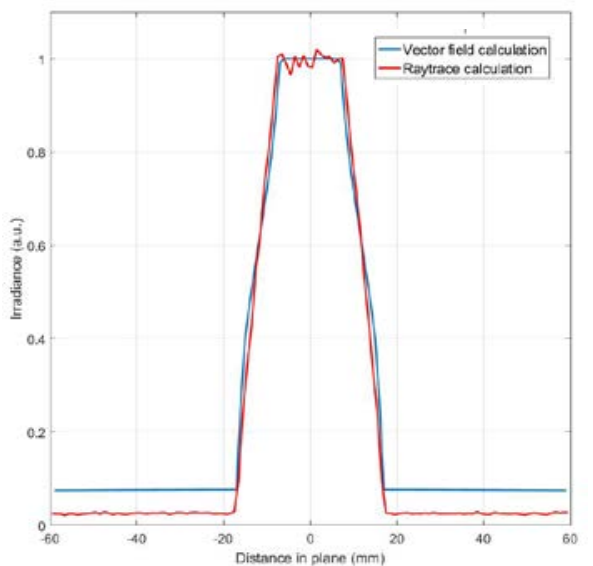

a)

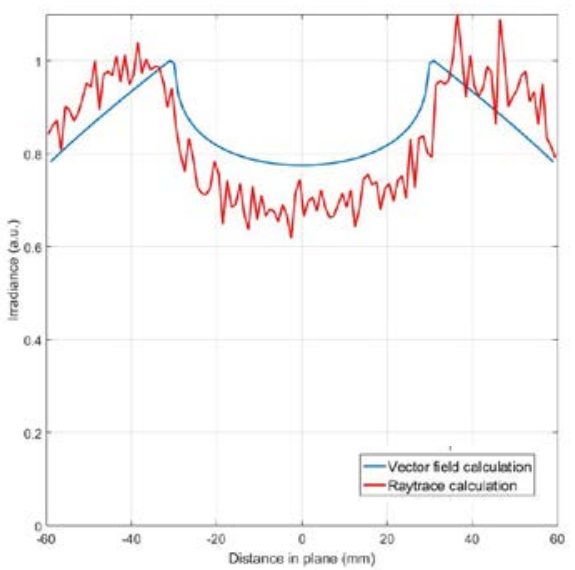

b)

Figure 9. Comparisons between vector field and raytrace calculations for reflector system 


\subsection{Computation time analysis}

One of the most relevant parameter to be consider in the analysis of this new technique to simulate optical systems, is the computation time. Nowadays, the present work is the first one on the technique of simulates optical systems using vector field calculations instead typical raytrace calculations, then the algorithms are not well optimized and the comparative is not well standardized. Nevertheless it is possible to study some basic differences between both calculations techniques. Raytrace technique typically uses vectorial forms of snell law and reflection law [**], and needs a great number of rays to reduce the noise in the detection plane. Then the computation time has typically a linear dependence with the number of optical interfaces and with number of rays. Vector field calculations only need a couple of rays but it use variational Fermat's principle, then the computation time is not dependence on number of rays but has an exponential dependence on the number of optical interfaces. Fortunately, optical systems typically use only a few optical interfaces, and then vector field calculation can maintain advantage on computation time analysis.

\section{VECTOR FIELD SIMULATION DEMO SOFTWARE}

Once we have showed the potential benefits to study optical systems using vector field calculations instead conventional raytrace analysis, and as we have mention. We start the task to build simulation software, to analyze the optical behavior of systems based on the calculus of irradiance vector field. We start that task using MATLAB software and our objective is to use GUI (Graphical User Interface) toolbox to provide and easy to use software interface. Of course this first version of the software includes big constraints, which will be removed step by step. First one is that simulation software only analyzes systems with a source, a lens and a detector. The dimensions of the source lens and detector are constrained too, in order to ensure the reliability of the software. With these constrains we have build an interface which includes fields to design the systems and three main capabilities, first one draw the system, second ones simulates the irradiance map at detector third simulates field lines in the space. Figure 10 shows the interface of the software, with three main parts. The upper part, fields to define the optical system including lens and reflection system, the left part, buttons to perform actions, the right part graphic to draw the system under study. Finally we have uploaded a free version of the VF_simulator in our webpage http://www.ucm.es/lightingandcolor.

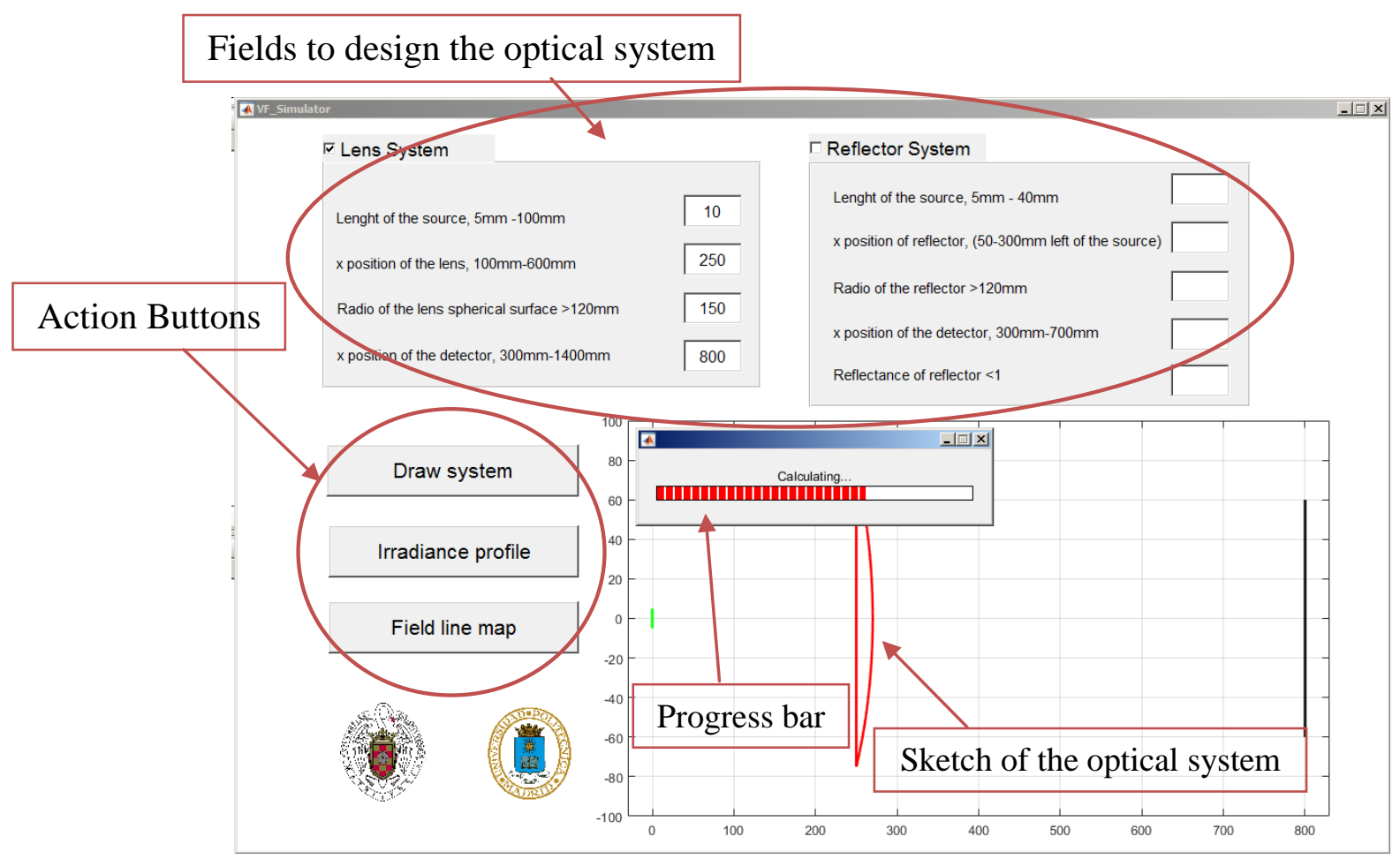

Figure 10. Interface of the Vector Field Simulation Software 


\section{CONCLUSIONS}

Theoretically we have shown the viability to employ irradiance vector field calculations instead conventional raytrace calculations. The advantages of use field vector are in computing time, in fact to calculate irradiance maps vector field technique needs only a few rays and raytrace needs millions rays, and in the information obtained. Field vector provides field line map, which inform us about the flow of energy by the system, reflector with the shape of field line and refractors orthogonal to field lines are ideal. We have develop an algorithm valid to compute field vector based on Fermat's principle, the field line allows the direction of the vector and field line density provides the modulus of the vector. We have proven this algorithm for standard configuration showing agreement between vector field calculations and raytrace calculations. Finally we have built a vector field simulation software, constrained to refractive or reflexive axisymmetric systems, we have upload this version of the software to our webpage for free download.

\section{REFERENCES}

[1] A. Gershun, “The light Field”, J. Math. Physics, vol. XVIII, 51-151, 1939.

[2] P. Moon, D.E. Spencer "Photic Field”, Massachusetts Institute of Technology Press, Massachusetts, 1981.

[3] J. T. Withrop, "Propagation of Structural Information in Optical Wave Fields", Journal of the Optical Society of America, 61 (1), 15-30, 1971.

[4] R. Winston, W.T. Welford "Geometrical vector flux and some new nonimaging concentrators", Journal of the Optical Society of America, 69 (4), 532-536, 1979.

[5] R. Winston, W.T. Welford "Ideal flux concentrators as shapes that do not disturb the geometrical vector flux field: A new derivation of the compound parabolic concentrator”, Journal of the Optical Society of America, 69 (4), 536-539, 1979.

[6] R. Winston, J. C. Miñano, P. Benitez, with contributions by N. Shatz, J. C. Bortz "Nonimaging Optics", Elsevier Academic Press, New York, 2005.

[7] A. García-Botella, A. A. Fernández-Balbuena, D. Vázquez, E. Bernabeu, A. González-Cano, "Hyperparabolic Concentrators”, Applied Optics, 48 (4), 712-715, 2009.

[8] V. Fock, “Zur Berechnung der Beleuchtungsstärke“, Zeitschrift für Physik, 28, 102-118, 1924.

[9] A. García-Botella, "Ideal flux field dielectric concentrators”, Applied Optics, 50 (28), 5357-5360, 2011.

[10] E. Hecht, “Optics”, Adison-Wesley, 2003. 